\title{
Correlation of serum amyloid A levels, clinical manifestations, treatment, and disease activity in patients with acute anterior uveitis
}

\author{
Ma-Li Dai ${ }^{1,2} \cdot$ Shipei $\mathrm{Fan}^{1,2} \cdot{\text { Zhuoran } \mathrm{Li}^{3} \cdot \text { Xuewen } \mathrm{Yu}^{1,2} \cdot{\text { Dan } \mathrm{Lin}^{1,2} \cdot \text { Xiu-Feng Huang }}^{1,2,4} \cdot \text { Yuqin Wang }}^{1,2}$
}

Received: 25 March 2019 / Revised: 5 October 2019 / Accepted: 14 October 2019 / Published online: 16 December 2019

(c) The Author(s), under exclusive licence to The Royal College of Ophthalmologists 2019

\begin{abstract}
Purpose To investigate the association between serum amyloid A (SAA) protein and the clinical features of acute anterior uveitis (AAU), and to evaluate the disease activity and treatment effect in relation to SAA levels.

Methods AAU patients and healthy individuals were recruited from October 2016 to August 2017 at the Department of Uveitis, in the Eye Hospital of Wenzhou Medical University. Related demographic, clinical characteristics, and therapeutic data were analyzed.

Results One hundred and eight AAU patients and 18 healthy controls were included in this study. Serum SAA levels in AAU patients were significantly higher than those of healthy controls $(p$ all $<0.0001)$. Significantly higher SAA levels were found in $\mathrm{AS}^{+} \mathrm{AAU}$ patients than those in $\mathrm{AS}^{-} \mathrm{AAU}$ patients $(p<0.05)$. SAA levels were also significantly higher in patients with HLA-B $27^{+}$AAU compared with those with HLA-B27 ${ }^{-}$AAU $(p<0.05)$. Furthermore, in each of the AAU subgroups, higher SAA levels were observed in the active state than those in the inactive state $(p$ all $<0.05)$. In addition, SAA levels were positively correlated to anterior chamber cell counts $(r=0.492, p<0.0001)$. ROC curve analysis revealed that SAA had an AUC value of 0.727 for detecting active inflammation (Youden's index $=0.38$ ). SAA decreased with effective treatments $(p=0.0002)$.

Conclusion Serum levels of SAA were elevated in AAU patients. The increased levels of SAA were correlated with AS and HLA-B27 status. SAA levels were also positively correlated to disease activity and decreased with effective treatments. These findings suggest that SAA is associated with AAU, with a potential role in monitoring inflammatory processes and assessing the efficacy of therapy.
\end{abstract}

These authors contributed equally: Ma-Li Dai, Shipei Fan

Xiu-Feng Huang

hxfwzmc@163.com

$\triangle$ Yuqin Wang

yqwang57@163.com

1 School of Ophthalmology and Optometry, Eye Hospital of Wenzhou Medical University, Wenzhou, Zhejiang, China

2 State Key Laboratory of Optometry, Ophthalmology, and Vision Science, Affiliated Eye Hospital, Wenzhou Medical University, Wenzhou, Zhejiang, China

3 New England College of Optometry, Boston, MA, USA

4 Translational Genomics Group, Institute of Health and Biomedical Innovation, Queensland University of Technology at Translational Research Institute, Princess Alexandra Hospital, Brisbane, QLD 4102, Australia

\section{Introduction}

Acute anterior uveitis (AAU) is a common inflammatory eye disease that mainly affects the iris and the anterior-ciliary body [1]. Typically, AAU patients present with a sudden onset of redness, pain, photophobia, and blurred vision, symptoms that usually last less than 3 months. Given that AAU primarily affects people between the age of 20 and 50 years, the personal and population burden are significant [2]. Human leucocyte antigen (HLA)-B27 is strongly associated with AAU. HLA-B27-positive AAU demonstrates much more significant cellular and fibrinous exudation into the aqueous humour, higher tendency for recurrences, and a much stronger association with ankylosing spondylitis (AS).

Serum amyloid A (SAA) is an acute-phase protein secreted in large quantities during acute inflammation [3, 4]. SAA induces the synthesis of TNF- $\alpha$, IL-1 $\beta$, IL- 6 , IL-17, as well as many other proinflammatory cytokines [4]. In Behcet's 
patients, SAA induces Th17 polarization rather than Th1 differentiation from CD4+ T cells [5]. It was found that SAA levels were significantly higher in Behcet's patients than those in healthy controls (HC) [6, 7], and that Behcet's patients with higher serum SAA levels showed higher levels of both serum pro-inflammatory markers and disease activity [8]. In Behcet's patients with SAA levels $>150 \mathrm{mg} / \mathrm{L}$ [9], SAA might be a biomarker indicative of ocular involvement and disease relapse. Elevation of serum SAA was also found in patients with AS, a disease known to be highly associated with AAU [10]. Another study speculated that SAA concentrations might be associated with AAU recurrence, but the data were from a small cohort of AAU patients, without considering the influence of AS [11]. In addition, Mitulescu et al. analyzed 11 AS patients presenting with AAU, and found that SAA levels correlated with both the number of AAU recurrences as well as with AS disease activity [12]. Despite these findings, the potential role and clinical significance of SAA in AAU still needs to be elucidated. In the present study, we aimed to investigate the association between SAA and AAU, as well as the disease activity and treatment effect.

\section{Materials and methods}

\section{Patients and clinical data}

Patient recruitment was performed at the Department of Uveitis, approved by the Ethic Committee of the Eye Hospital of Wenzhou Medical University. Consecutive patients with AAU were recruited from October 2016 to August 2017. All enroled patients signed an informed consent and agreed to participate in the study. All of the recruited patients and assessment criteria met the Standardization of Uveitis Nomenclature (SUN) Guidelines. AAU was defined as an attack of uveitis, mainly affecting the iris and the anterior-ciliary body, with a sudden onset and limited duration. Patients who had previously been diagnosed with AAU in our Department, but who had inactive disease at the time of recruitment, were also included in this study. Active AAU patients were treated with topical steroids, cycloplegics, and nonsteroidal anti-inflammatory eyedrops. Because young people were less likely to suffer from diseases, 18 healthy individuals were enroled as HCs.

Detailed information about the history of the enroled patients was collected. Clinical characteristics including age at recruitment, gender, age of onset of initial attack, recurrence, slit-lamp examination features, HLA-B27 subtype status, and history of systematic diseases, such as AS, were collected. The definitive diagnosis of AS was made by a rheumatologist according to the 1984 modified New York criteria. Patients were excluded if (1) the HLA-B27 test or AS status was not available; (2) they had a viral, bacterial, tuberculin, or syphilitic aetiology; (3) the results of TORCH test indicated IgM positivity; (4) they exhibited another uveitis subtype, such as Fuchs heterochromic iridocyclitis, Posner-Schlossman syndrome, Behcet's disease, or Vogt-Koyanagi-Harada syndrome; (5) AAU was resulted from trauma; (6) they suffered from angiocardiopathy, diabetes mellitus, obesity, tumour, amyloidosis, or other autoinflammatory diseases; (7) they had received medications or dietary supplements within the previous month.

\section{Sample collection and analysis of SAA}

Blood samples of included patients were obtained by Vacutainer EDTA tubes within $2 \mathrm{~h}$ after the ophthalmologist's examination. The samples were centrifuged at $1500 \mathrm{~g}$ for $10 \mathrm{~min}$ within $1 \mathrm{~h}$ after acquisition. Within $2 \mathrm{~h}$ of phlebotomy, plasma was separated for analysis and SAA levels were measured using a PMDT800 immunochromatography analyzer (Pro-med Tech Ltd, Beijing, China). The lowest detection limit for SAA was $1 \mathrm{mg} / \mathrm{L}$, hence for comparison purposes in this study, a level of $<1 \mathrm{mg} / \mathrm{L}$ was standardized as $0 \mathrm{mg} / \mathrm{L}$.

\section{Statistical analysis}

Data analysis was performed using SPSS V22.0. All data in this study were expressed using percentiles, and were shown as median ( $25 \%$ percentile, $75 \%$ percentile). The independent or paired $t$-tests were used to detect differences when parametric analysis was possible. If it was not possible, the Mann-Whitney $U$-test was employed instead. A $p$ value less than 0.05 was deemed as statistically significant. Spearman correlation analysis was used to evaluate the correlation between anterior chamber cell numbers and the SAA level. A multiple linear regression was performed to evaluate the possible effects of factors on the SAA level.

\section{Results}

\section{General information}

In total, 161 AAU patients agreed to participate in our study. Among them, 53 patients were excluded based on the exclusion criteria. Thus, a total of 108 AAU patients were recruited and evaluated in the study (Table 1). At the time of serum collection, 57 patients presented with active AAU and 51 patients had inactive disease. Of the active AAU patients, 12 agreed to provide more than one blood sample, enabling collection when their inflammation was in remission after discontinuing treatment. The data from these 12 active AAU patients were selected for a separate analysis. Of the $18 \mathrm{HCs}$ enroled, 11 were men and 7 were women, with an average age of $29.5 \pm 8.3$ years. 
Table 1 Demographic and clinical data of active and inactive AAU patients.

\begin{tabular}{llll}
\hline & AAU & Active & Inactive \\
\hline Male/female; $n$ & $108(59 / 49)$ & $57(35 / 22)$ & $51(24 / 27)$ \\
AS $^{+} / \mathrm{AS}^{-} ; n$ & $47 / 61$ & $27 / 30$ & $20 / 31$ \\
HLA-B27 positivity; $n(\%)$ & $83(76.9 \%)$ & $46(80.7 \%)$ & $37(72.5 \%)$ \\
Age, years; median (Q1, Q3) & $41.5(33,49)$ & $40(32.5,46)$ & $44(35,51)$ \\
Onset age, years; median (Q1, Q3) & $36(29,45)$ & $34(29.5,43.5)$ & $36.5(28.3,47.8)$ \\
Recurrence episodes; median (Q1, Q3) & $2(1,3)$ & $2(1,3)$ & $2(1,3)$ \\
\hline
\end{tabular}

Median $(Q 1, Q 3)$ median (25\% percentile, $75 \%$ percentile)

$A A U$ acute anterior uveitis, $A S$ ankylosing spondylitis, $H L A-B 27$ human leucocyte antigen-B27, $n$ number
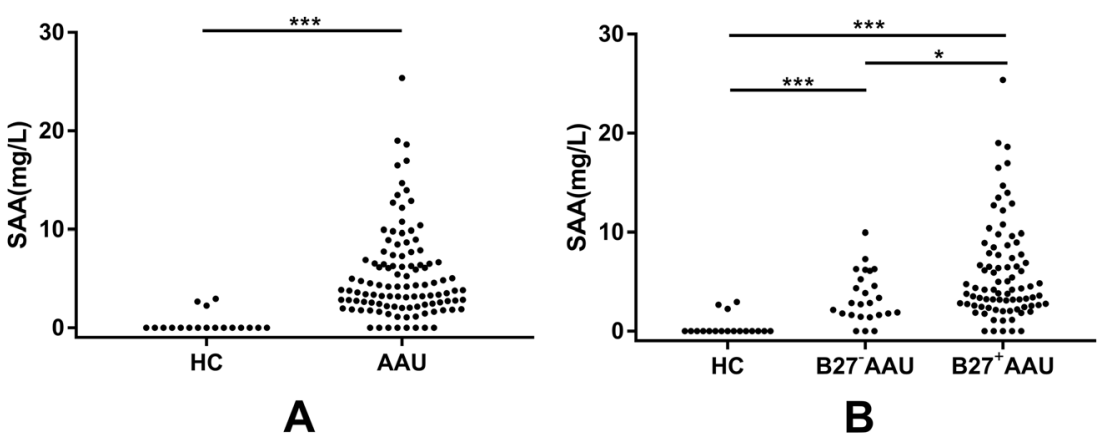

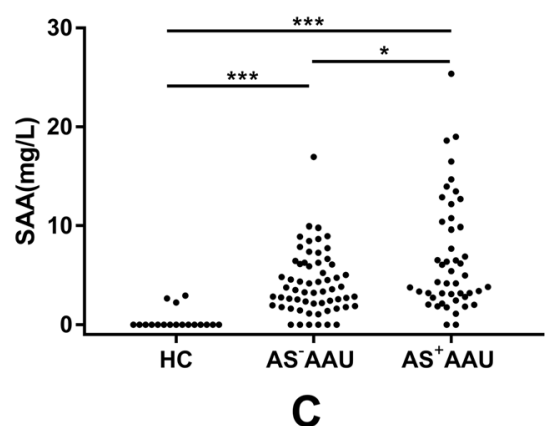

HLA-B27-negative AAU, B27 ${ }^{+}$AAU HLA-B27-positive AAU, AS ${ }^{-}$ AAU AAU without AS, $\mathrm{AS}^{+} \mathrm{AAU}$ AAU with AS, HC healthy controls. All $p$ values were calculated using the Mann-Whitney $U$-test.
Fig. 1 SAA levels in AAU. SAA levels were measured and compared in a AAU patients and $\mathrm{HC}$; b B27- AAU patients, B27 $7^{+} \mathrm{AAU}$ patients, and $\mathrm{HC}$; $\mathrm{c}^{-} \mathrm{AAU}$ patients, $\mathrm{AS}^{+} \mathrm{AAU}$ patients, and $\mathrm{HC} . * p<0.05$, $* * p<0.01, * * * p<0.001$, AAU acute anterior uveitis, $\mathrm{B} 27^{-} \mathrm{AAU}$
Table 2 Comparison of SAA levels in different groups.

\begin{tabular}{llllc}
\hline Comparison & $n$ & Median (Q1, Q3) of SAA level $(\mathrm{mg} / \mathrm{L})$ & $U$ value & $P$ value \\
\hline HC vs. AAU & 18 vs. 108 & $0(0,0)$ vs. $3.83(2.22,6.83)$ & 159 & $<0.0001$ \\
Active vs. inactive AAU & 57 vs. 51 & $5.42(3.25,9.83)$ vs. $2.81(1.78,4.98)$ & 794.5 & $<0.0001$ \\
$\mathrm{HLA}^{-B} 27^{-}$vs. HLA B27 & 25 vs. 83 & $2.83(1.63,5.67)$ vs. $4.19(2.59,7.87)$ & 703 & 0.014 \\
$\mathrm{AS}^{-}$vs. $\mathrm{AS}^{+}$ & 61 vs. 47 & $3.51(1.92,6.20)$ vs. $4.31(2.81,10.87)$ & 1071 & 0.024 \\
\hline
\end{tabular}

Calculated using Mann-Whitney $U$-test; Median $(Q 1, Q 3)$ Median (25\% percentile, $75 \%$ percentile) $n$ number

\section{SAA levels in AAU Patients}

SAA levels were analyzed in 108 AAU patients and 18 HCs. The mean SAA level (all data were shown as median (25\% percentile, $75 \%$ percentile)) was much lower in the HC group $(0(0,0))$ than in the AAU patients $(3.83(2.22$, 6.83), $p<0.0001$, Fig. 1a and Table 2).

SAA levels were then analyzed in AAU patients according to HLA-B27 status (Fig. 1b). We observed that the SAA levels in patients with HLA-B27-positive AAU $\left(\mathrm{HLA}-\mathrm{B} 27^{+} \mathrm{AAU}\right)$ were significantly higher than patients with HLA-B27-negative AAU (HLA-B27- AAU) (4.19 $(2.59,7.87)$ vs. $2.83(1.63,5.67), p=0.014)$ (Table 2$)$. Both HLA-B $27^{+}$AAU and HLA-B $27^{-}$AAU patients displayed increased SAA levels when compared with the HC group ( $p$ both $<0.0001$ ) (Fig. 1b).
We then carried out further stratified analysis for AAU patients with or without AS (Fig. 1c). Notably, we found greater elevation of SAA in AAU patients with AS $\left(\mathrm{AS}^{+} \mathrm{AAU}\right)$ than those without $\mathrm{AS}$ ( $\left.\mathrm{AS}^{-} \mathrm{AAU}\right)$ (4.31 $(2.81,10.87)$ vs. $3.51(1.92,6.20), p=0.024)$ (Table 2$)$. In addition to this finding, we observed significant differences in $\mathrm{AS}^{+} \mathrm{AAU}$ and $\mathrm{AS}^{-} \mathrm{AAU}$ patients when compared with the HC group (Fig. 1c, $p$ both $<0.0001$ ).

\section{SAA levels associated with disease activity in AAU Patients}

We then investigated the association between SAA level and disease activity. Increased SAA levels were observed in active state compared with inactive state of AAU patients $(5.42(3.25,9.83)$ vs. $2.81(1.78,4.98), p<0.0001)$ (Table 2 

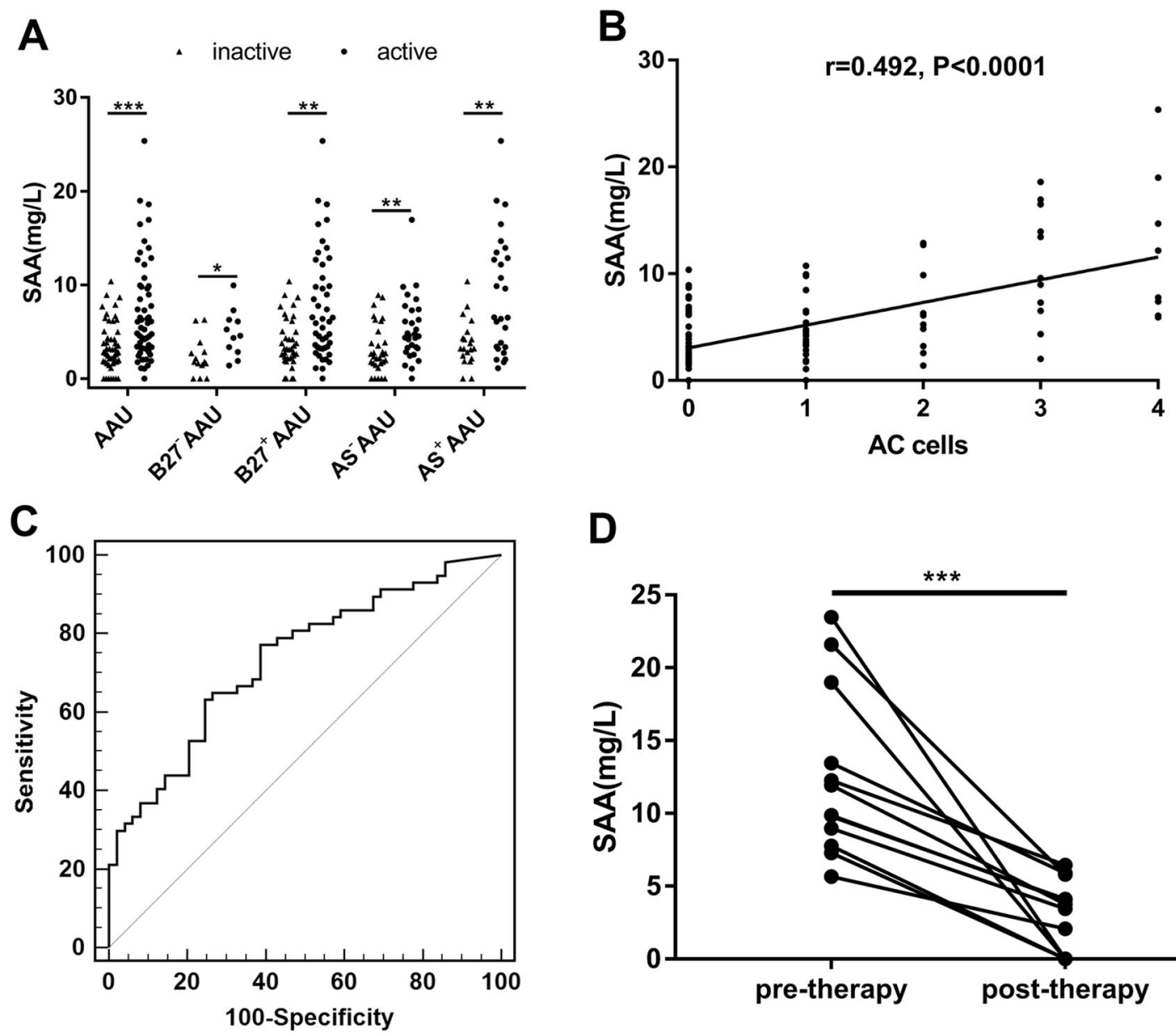

D

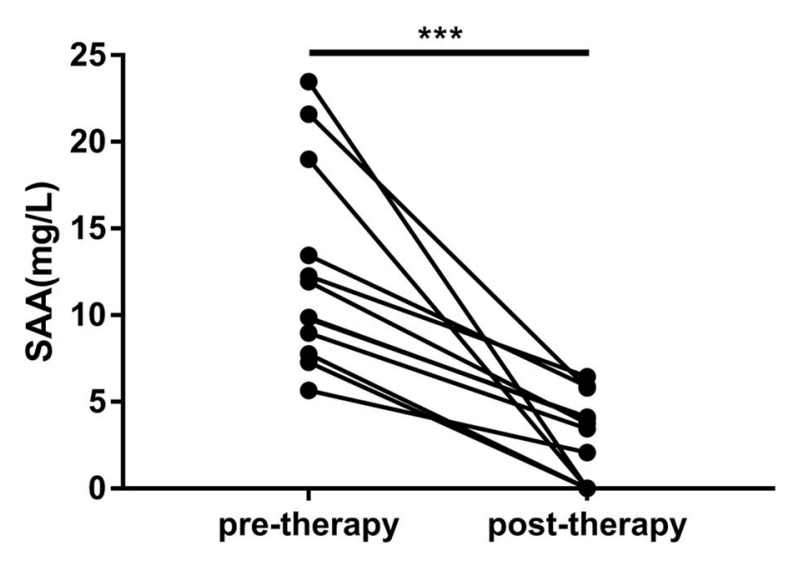

Fig. 2 Associations between SAA and disease activity and AC cells and treatment. a The comparison of SAA levels between active and inactive status in $\mathrm{AAU}, \mathrm{B} 27^{-} \mathrm{AAU}, \mathrm{B} 27^{+} \mathrm{AAU}, \mathrm{AS}^{-} \mathrm{AAU}$, and $\mathrm{AS}^{+} \mathrm{AAU}$ patients. b The correlation between SAA levels and $\mathrm{AC}$ cells was determined by Spearman rank correlation analysis. c The ROC curve for detecting the active inflammation of AAU by SAA.

d SAA levels in AAU patients before and after therapy were compared using paired $t$-test. $* p<0.05, * * p<0.01, * * * p<0.001$, AAU acute anterior uveitis, B27- AAU HLA-B27-negative AAU, B27 ${ }^{+}$AAU HLA-B27-positive AAU, $\mathrm{AS}^{-} \mathrm{AAU}$ AAU without $\mathrm{AS}, \mathrm{AS}^{+} \mathrm{AAU}$ AAU with AS, AC cells anterior chamber cells.

and Fig. 2a). The same association trend between SAA levels and disease activity was also observed in HLA-B27 AAU patients $(4.57(2.83,6.28)$ vs. $1.78(1.07,3.09), p<$ $0.05)$, HLA-B $27^{+}$AAU patients $(6.00(3.26,11.09)$ vs.3.19 $(2.17,5.89), p<0.01), \mathrm{AS}^{-} \mathrm{AAU}$ patients $(4.55(3.12,7.31)$ vs. $2.59(1.62,5.04), p<0.01)$, and $\mathrm{AS}^{+} \mathrm{AAU}$ patients $(6.52$ $(3.24,13.45)$ vs. $3.20(2.23,4.81), p<0.01)$.

As the grading of anterior chamber cells (AC cells) based on the SUN Guidelines was critical for evaluating the clinical stage of AAU, we aimed to investigate the association between SAA levels and AC cells. We observed significant positive correlation between SAA levels and AC cells among these 108 AAU patients (Fig. 2b, $r=0.492$, $p<0.001)$. SAA levels were assessed to detect the active inflammation of AAU by ROC curve (Fig. 2c). The area under the ROC curve (AUC) obtained for the SAA levels was 0.727 (cutoff value $=3.22 \mathrm{mg} / \mathrm{L}$, Youden's index $=$ 0.38 , sensitivity $=77.2 \%$, specificity $=60.8 \%$ ).

A follow up study was conducted in which SAA levels among 12 patients were tracked from active to inactive inflammatory state after treatment during an attack. The results showed that the SAA level decreased significantly along with the improvement of AAU inflammation after treatment (Fig. 2 d, from $10.9(8.06,17.61)$ to $3.61(0,5.38)$ $\mathrm{mg} / \mathrm{L}, p=0.0002)$.

\section{Multiple linear regression analysis}

We used a multiple linear regression model analysis to assess the factors contributing to elevated SAA levels (Table 3). In the regression analysis, recurrence $(p=0.026$, $95 \% \mathrm{CI}=-0.808,-0.052)$, concurrence with AS $(p=$ $0.033,95 \% \mathrm{CI}=0.162,3.730)$, anterior chamber cell counts $(p<0.001,95 \% \mathrm{CI}=1.466,2.685)$ all conform to the model as the predicting parameters for the SAA level $\left(R^{2}=0.476\right.$, Table 3$)$. We calculated standardized $\beta$ in 
Table 3 Multiple linear regression of predicted factors for the SAA level.

\begin{tabular}{lllll}
\hline & $B$ coefficient & $95 \%$ CI & $\beta$ coefficient & $p$ value \\
\hline Constant & 2.252 & $-1.392,5.895$ & - & 0.223 \\
Gender & 0.417 & $-1.239,2.074$ & 0.042 & 0.618 \\
Recurrence & -0.430 & $-0.808,-0.052$ & -0.184 & $0.026^{*}$ \\
Onset age & 0.001 & $-0.071,0.074$ & 0.003 & 0.968 \\
HLA-B27 & 1.170 & $-0.902,3.243$ & 0.100 & 0.265 \\
Ankylosing spondylitis & 1.946 & $0.162,3.730$ & 0.193 & $0.033^{*}$ \\
Anterior chamber cells & 2.075 & $1.466,2.685$ & 0.555 & $<0.001^{*}$ \\
\hline
\end{tabular}

$B$ unstandardized coefficient; $\beta$ standardized coefficient

$C I$ confidence interval

${ }^{*} p$ value is $<0.05$ order to evaluate the most significant parameter for SAA level. The results revealed that the SAA level was most positively affected by anterior chamber cells. Gender, onset age, and HLA-B27 status were not significantly associated with SAA levels.

\section{Discussion}

SAA is one of the major nonspecific acute-phase proteins, whose concentration in circulating blood significantly increases during acute inflammation. The liver is considered to be the major site of SAA synthesis. In addition, SAA synthesis is known to occur in many other cells and tissues, including macrophages, kidneys, lungs, adipocytes, and mammary glands $[13,14]$. Expression of SAA mRNA has also been detected in the fibrovascular membranes of patients with proliferative diabetic retinopathy as well as in the trabecular meshwork of patients with glaucoma $[15,16]$. We speculated that, in patients with AAU, SAA might be synthesized in the iris, although, further laboratory investigations will be required to verify the origin of SAA in AAU patients.

Several studies have reported the association between SAA and other autoimmune and inflammation diseases, such as Rheumatoid arthritis [17, 18], AS [12, 19], juvenile idiopathic arthritis [20, 21], and Behcet's disease [8, 9, 22]. Multiple studies have shown that AAU is frequently associated with AS. For example, HLA-B27 and endoplasmic reticulum aminopeptidases-1 (ERAP-1) are both associated with AAU and AS [23, 24]. In our study cohort, 47/108 of patients had both AAU and AS. We carried out a stratified analysis for AAU patients with or without AS, and found that SAA levels were significantly elevated in both $\mathrm{AS}^{+}$ $\mathrm{AAU}$ and $\mathrm{AS}^{-} \mathrm{AAU}$ patients, than in healthy individuals. It is worth noting that the significantly higher SAA levels in $\mathrm{AS}^{-} \mathrm{AAU}$ patients demonstrated that elevated SAA levels were a result of AAU inflammation. Meanwhile, the SAA levels of $\mathrm{AS}^{+} \mathrm{AAU}$ patients were significantly higher than those in $\mathrm{AS}^{-} \mathrm{AAU}$ patients. These data indicated that the concentration of SAA in AAU patients was also associated with AS status.

HLA-B27 is highly associated with AAU, and HLA-B27 ${ }^{+}$ AAU exhibited distinctive clinical features when compared with HLA-B27- AAU [25]. Typical manifestations of AAU patients, especially those with HLA-B $27^{+} \mathrm{AAU}$, were characterized by severe ocular inflammation such as fibrin and hypopyon in the anterior chamber. The results of this study demonstrated that HLA-B $27^{+}$AAU patients had higher SAA levels than HLA-B27 ${ }^{-}$AAU patients, a finding that is consistent with these disease subsets either having different disease activity or pathogenesis. Previous studies have indicated that HLA-B27 may impact the gut and/or the eye itself, where loss of tolerance could contribute to uveitogenesis [26]. Rosenbaum et al. proposed several models focusing on the intestinal microbiome as a key component of AAU pathogenesis. Professor Peizeng Yang revealed significant alterations in microbiota metabolism in AAU patients compared with HCs, following the analysis of PCoA plots of unweighted UniFrac distances [27]. Segmented filamentous bacteria were reported to made direct contact with the ileum epithelium and induce SAA proteins, thereby promoting IL17A expression in ROR $\gamma \mathrm{t}+\mathrm{T}$ cells [28].

In the current study, we recruited a sizable AAU cohort with detailed clinical recording. We comprehensively investigated the association between AAU and SAA. A previous study has reported that the severity of uveitis was equal in AAU patients with elevated SAA and normal SAA [11]. However, this study did not consider the effect of AS. In this research, we observed increased SAA levels in the active state than those in the inactive state of each subgroup. Furthermore, there was a significant positive correlation between SAA levels and anterior chamber cell counts. SAA levels decreased significantly as AAU inflammation diminished with effective treatment.

This study does, however, have several limitations. First, this clinical research explored the association between SAA and the clinical manifestations of AAU, such as disease 
activity, among a cohort of 108 patients with AAU. The pathogenic mechanism of SAA's involvement in AAU is unclear and needs to be elucidating by further laboratory investigation. Second, by conducting the research in a uveitis clinic, this study may have been biased towards patients with recurrent or more severe AAU. Third, we observed that recurrence is associated with lower levels of SAA, which was also reported by a study by Yorston et al. [11]. At this point, however, we do not have an explanation for this. One possible cause is that the performance of our multiple linear regression model is moderate $\left(R^{2}=0.476\right)$. Further experiments should be performed to address this issue.

In summary, our study showed that serum levels of SAA were elevated in patients with AAU, and that these increased SAA levels were correlated with AS and HLAB27 status. SAA levels were also positively correlated to disease activity and decreased with effective treatments. The results of this study suggest that SAA is associated with AAU and identify a potential avenue for monitoring the inflammatory processes during diagnosis and treatment, as well as assessing the efficacy of therapy.

\section{Summary}

\section{What was known before}

- SAA is one of the major nonspecific acute-phase proteins, whose concentration in blood circulation significantly increases during acute inflammation.

- Several studies have reported the association between SAA and other autoimmune and inflammation diseases, such as Rheumatoid arthritis, ankylosing spondylitis, juvenile idiopathic arthritis, and Behcet's disease.

\section{What this study adds}

- SAA levels significantly increased in AAU patients than those in $\mathrm{HC}$, and these differences were also detected in HLA-B $27^{+}$AAU, HLA-B27 ${ }^{-}$AAU, AS $^{+}$AAU and AS $^{-}$ AAU.

- SAA levels were significantly correlated with disease activity and treatment in patients with AAU.

- Multiple linear regression model analysis demonstrated that recurrence, concurrence with AS, and more anterior chamber cells were associated with elevation of SAA level.

Acknowledgements We would like to thank all of the donors that participated in the present study. This study was supported by the
Innovation Research Program of the Eye Hospital (YNCX201608), National Natural Science Foundation of China (31771390), Key Research Program of the Eye Hospital (YNZD201402), Wenzhou Science and Technology Foundation (Y20160457), General Program of the Eye Hospital (YNKT201601), National Students' Innovation Training Program (201810343005), XinMiao Talents Program of Zhejiang Province (2018R413036).

\section{Compliance with ethical standards}

Conflict of interest The authors declare that they have no conflict of interest.

Publisher's note Springer Nature remains neutral with regard to jurisdictional claims in published maps and institutional affiliations.

\section{References}

1. Jabs DA, Nussenblatt RB, Rosenbaum JT, Standardization of Uveitis Nomenclature Working Group Standardization of uveitis nomenclature for reporting clinical data. Results of the First International Workshop. Am J Ophthalmol. 2005;140:509-16.

2. Chang JH, Wakefield D. Uveitis: a global perspective. Ocul Immunol Inflamm. 2002;10:263-79.

3. Eklund KK, Niemi K, Kovanen PT. Immune functions of serum amyloid A. Crit Rev Immunol. 2012;32:335-48.

4. Ye RD, Sun L. Emerging functions of serum amyloid $A$ in inflammation. J Leukoc Biol. 2015;98:923-9.

5. Lucherini OM, Lopalco G, Cantarini L, Emmi G, Lopalco A, Venerito V, et al. Critical regulation of Th17 cell differentiation by serum amyloid-A signalling in Behcet's disease. Immunol Lett. 2018;201:38-44.

6. Aygunduz M, Bavbek N, Ozturk M, Kaftan O, Kosar A, Kirazli S. Serum beta 2-microglobulin reflects disease activity in Behcet's disease. Rheumatol Int. 2002;22:5-8.

7. Mao L, Dong $\mathrm{H}$, Yang $\mathrm{P}$, Zhou $\mathrm{H}$, Huang $\mathrm{X}$, Lin $\mathrm{X}$, et al. MALDI-TOF/TOF-MS reveals elevated serum haptoglobin and amyloid A in Behcet's disease. J Proteome Res. 2008;7:4500-7.

8. Lopalco G, Lucherini OM, Vitale A, Talarico R, Lopalco A, Galeazzi M, et al. Putative role of serum amyloid-A and proinflammatory cytokines as biomarkers for Behcet's Disease. Medicine. 2015;94:e1858.

9. Sota J, Vitale A, Rigante D, Orlando I, Lucherini OM, Simpatico A, et al. Correlation of serum amyloid-A levels, clinical manifestations, treatment, and disease activity in patients with Behcet's Disease. Isr Med Assoc J. 2018;20:517-21.

10. Londono J, Romero-Sanchez MC, Torres VG, Bautista WA, Fernandez DJ, Quiroga Jde A, et al. The association between serum levels of potential biomarkers with the presence of factors related to the clinical activity and poor prognosis in spondyloarthritis. Rev Bras Reumatol. 2012;52:536-44.

11. Yorston D, Whicher J, Chambers R, Klouda P, Easty D. The acute phase response in acute anterior uveitis. Trans Ophthalmol Soc UK. 1985;104:166-70.

12. Mitulescu TC, Stavaru C, Voinea LM, Banica LM, Matache C, Predeteanu D. The role of vitamin D in immuno-inflammatory responses in ankylosing spondylitis patients with and without acute anterior uveitis. J Med Life. 2016;9:26-33.

13. Sack GH Jr., Zachara N, Rosenblum N, Talbot CC Jr., Kreimer S, Cole R, et al. Serum amyloid A1 (SAA1) protein in human colostrum. FEBS Open Bio. 2018;8:435-41.

14. Urieli-Shoval S, Cohen P, Eisenberg S, Matzner Y. Widespread expression of serum amyloid A in histologically normal human 
tissues. Predominant localization to the epithelium. J Histochem Cytochem. 1998;46:1377-84.

15. Ma Y, Tao Y, Lu Q, Jiang YR. Intraocular expression of serum amyloid a and interleukin- 6 in proliferative diabetic retinopathy. Am J Ophthalmol. 2011;152:678-685 e672.

16. Wang WH, McNatt LG, Pang IH, Hellberg PE, Fingert JH, McCartney MD, et al. Increased expression of serum amyloid A in glaucoma and its effect on intraocular pressure. Investig Ophthalmol Vis Sci. 2008;49:1916-23.

17. Hwang YG, Balasubramani GK, Metes ID, Levesque MC, Bridges SL Jr., Moreland LW. Differential response of serum amyloid A to different therapies in early rheumatoid arthritis and its potential value as a disease activity biomarker. Arthritis Res Ther. 2016;18:108

18. Shen C, Sun XG, Liu N, Mu Y, Hong CC, Wei W, et al. Increased serum amyloid $\mathrm{A}$ and its association with autoantibodies, acute phase reactants and disease activity in patients with rheumatoid arthritis. Mol Med Rep. 2015;11:1528-34.

19. Jung SY, Park MC, Park YB, Lee SK. Serum amyloid a as a useful indicator of disease activity in patients with ankylosing spondylitis. Yonsei Med J. 2007;48:218-24.

20. Cantarini L, Giani T, Fioravanti A, Iacoponi F, Simonini G, Pagnini I, et al. Serum amyloid A circulating levels and disease activity in patients with juvenile idiopathic arthritis. Yonsei Med J. 2012;53:1045-8.

21. Bauer D, Kasper M, Walscheid K, Koch JM, Muther PS, Kirchhof $\mathrm{B}$, et al. Multiplex cytokine analysis of aqueous humor in juvenile idiopathic arthritis-associated anterior uveitis with or without secondary glaucoma. Front Immunol. 2018;9:708.

22. Lee HJ, Kim JH, Kim SW, Joo HA, Lee HW, Kim YS, et al. Proteomic analysis of serum amyloid A as a potential marker in intestinal Behcet's Disease. Dig Dis Sci. 2017;62:1953-62.

23. Robinson PC, Claushuis TA, Cortes A, Martin TM, Evans DM, Leo $\mathrm{P}$, et al. Genetic dissection of acute anterior uveitis reveals similarities and differences in associations observed with ankylosing spondylitis. Arthritis Rheumatol. 2015;67: $140-51$.

24. Robinson PC, Leo PJ, Pointon JJ, Harris J, Cremin K, Bradbury LA, et al. The genetic associations of acute anterior uveitis and their overlap with the genetics of ankylosing spondylitis. Genes Immun. 2016;17:46-51.

25. Wakefield D, Yates W, Amjadi S, McCluskey P. HLA-B27 anterior uveitis: immunology and immunopathology. Ocul Immunol Inflamm. 2016;24:450-9.

26. Rosenbaum JT, Asquith M. The microbiome and HLA-B27associated acute anterior uveitis. Nat Rev Rheumatol. 2018;14: 704-13.

27. Huang X, Ye Z, Cao Q, Su G, Wang Q, Deng J, et al. Gut microbiota composition and fecal metabolic phenotype in patients with acute anterior uveitis. Investig Ophthalmol Vis Sci. 2018;59:1523-31.

28. Sano T, Huang W, Hall JA, Yang Y, Chen A, Gavzy SJ, et al. An IL-23R/IL-22 circuit regulates epithelial serum amyloid A to promote local effector Th17 responses. Cell. 2015;163:381-93. 